\title{
Combined Use of Neutrophil to Lymphocyte Ratio and C-Reactive Protein Level to Predict Clinical Outcomes in Acute Myocardial Infarction Patients Undergoing Percutaneous Coronary Intervention
}

\author{
Ho-Cheol Shin, MD, Jae-Sik Jang, MD, Han-Young Jin, MD, Jeong-Sook Seo, MD, Tae-Hyun Yang, MD, \\ Dae-Kyeong Kim, MD, and Dong-Soo Kim, MD \\ Department of Internal Medicine, Busan Paik Hospital, Inje University College of Medicine, Busan, Korea
}

Background and Objectives: Both neutrophil to lymphocyte ratio (NLR) and C-reactive protein (CRP) are biomarkers associated with poor prognosis of patients with acute myocardial infarction (AMI). However, the combined usefulness of NLR and CRP in predicting adverse outcomes has not been investigated.

Subjects and Methods: We analyzed 381 consecutive AMI patients who underwent percutaneous coronary intervention (PCI) from January 2012 to January 2014. The endpoints were all-cause mortality, recurrent myocardial infarction (MI), stent thrombosis, repeat revascularization, stroke, and major adverse cardiac and cerebrovascular events (MACCE) at 2-year follow-up. Included patients were divided into 4 groups according to the optimal cut-off values for NLR and CRP on receiver operating characteristic analysis predicting mortality.

Results: Patients with both high NLR (>6.30) and high CRP (>0.76) had significantly greater risk of all-cause death and MACCE at 24 months, with no significant increase in the risk of recurrent $\mathrm{Ml}$, stent thrombosis, or stroke compared with patients with either low NLR or low CRP, as well as those with low NLR and low CRP. Kaplan-Meier analysis revealed significantly lower survival in patients with high NLR-CRP. On Cox multivariate analysis, high NLR-CRP (hazard ratio 23.172, 95\% confidence interval 6.575 to 81.671, $p<0.001$ ) was an independent predictor of all-cause death.

Conclusion: Elevated levels of both NLR and CRP are associated with increased risk of long-term mortality in AMI patients who have undergone PCl. (Korean Circ J 2017;47(3):383-391)

KEY WORDS: Neutrophils; Lymphocytes; Myocardial Infarction; Prognosis.

\section{Introduction}

Previous studies have shown that inflammatory response plays an important role in the progression and destabilization of

Received: September 13, 2016

Revision Received: December 9, 2016

Accepted: December 30, 2016

Correspondence: Jae-Sik Jang, MD, Department of Internal Medicine, Busan Paik Hospital, Inje University College of Medicine, 75 Bokji-ro, Busanjin-gu, Busan 47392, Korea

Tel: 82-51-890-6418, Fax: 82-51-890-6341

E-mail: jsjang71@gmail.com

- The authors have no financial conflicts of interest.

This is an Open Access article distributed under the terms of the Creative Commons Attribution Non-Commercial License (http://creativecommons.org/ licenses/by-nc/3.0) which permits unrestricted non-commercial use, distribution, and reproduction in any medium, provided the original work is properly cited. atherosclerosis and cardiovascular disease (CVD). ${ }^{12)}$ Among the various inflammatory markers related to CVD neutrophil to lymphocyte ratio (NLR) and C-reactive protein (CRP) have recently been investigated as new predictors of worse cardiovascular outcomes. ${ }^{3 / 4)}$ NLR is an inexpensive, easily obtainable, and widely available inflammatory biomarker associated with CVD. High NLR is an important predictor of mortality in patients with acute coronary syndrome, and it also has been reported as a prognostic marker for poor outcomes of coronary artery bypass graft surgery and postoperative atrial fibrillation. ${ }^{56)} \mathrm{CRP}$ is an inflammatory biomarker and is known as an independent predictor of adverse cardiovascular events in coronary artery disease (CAD) patients. ${ }^{3)}$ Although both NLR and CRP could be used as potential predictors of poor prognosis in acute myocardial infarction (AMI) patients, the combined usefulness of NLR and CRP in the prediction of adverse outcomes has not been investigated. Moreover, the combination of 2 biomarkers may increase the predictive ability of individual markers in AMI patients with multiple comorbidities. In previous studies, 
performed in other disease entities such as cancers, the combined use of NLR and CRP level was found to be helpful for predicting survival."7) It was found that patients with both an elevated CRP level and high NLR had a poorer survival rate than those with either an elevated CRP level or high NLR.

We therefore examined whether the combined parameter of NLR and CRP is associated with clinical outcomes and could be used as a useful predictor of long-term adverse events in AMI patients undergoing percutaneous coronary intervention (PCI).

\section{Subjects and Methods}

\section{Study subjects}

All consecutive eligible AMI patients between January 2012 and January 2014 who underwent successful PCI were retrospectively enrolled in this study. Exclusion criteria were; 1) patients older than 80 years, 2) patients with systemic disease, malignancy, inflammatory disease, acute infectious disease, severe renal disease (serum creatinine $\geq 10 \mathrm{mg} / \mathrm{dL}$ ) or liver disease, and 3) patients taking medications that have an effect on white blood cell (WBC) count. ST-elevation myocardial infarction (STEMI) was defined as the characteristic symptom of myocardial ischemia in association with persistent ST-elevation on electrocardiography and subsequent release of biomarkers of myocardial necrosis. ${ }^{8)}$ Non-STelevation myocardial infarction (NSTEMI) was defined by positive biomarkers of necrosis in the absence of ST-segment elevation in an appropriate clinical setting (chest discomfort or angina equivalent).9) This study was approved by the Institutional Review Board of Inje University Busan Paik Hospital and was conducted in accordance with the Declaration of Helsinki.

\section{Study endpoints and definitions}

The endpoints of this study were all-cause death, cardiovascular death, recurrent myocardial infarction (MI), repeat revascularization, stent thrombosis, stroke, and major adverse cardiac and cerebrovascular events (MACCE) within 24 months from the index PCI. All-cause death was defined as mortality from any cause. Cardiovascular death was defined as death from any cardiac cause or sudden unexpected death. Stroke was defined according to the Academic Research Consortium. ${ }^{10)}$ Repeat revascularization included any coronary procedures including target vessel revascularization and target lesion revascularization.

\section{Laboratory examinations and medications}

Hemoglobin, liver enzymes (aspartate aminotransferase, alanine transaminase), lipid profiles (total cholesterol, high-density lipoproteincholesterol, low-density lipoprotein [LDL]-cholesterol, triglycerides), serum glucose, hemoglobin A1c, and serum creatinine levels were measured at admission. Complete blood count (CBC), which includes total WBC count with profile, and platelet count and CRP value were assessed shortly before the index PCI. CBC was performed with an automated blood cell counter (XE-200, Sysmex, Kobe, Japan), and CRP was measured by immunoturbidimetric method (Toshiba 200FR Neo, Toshiba Medical Systems Co., Tochigi-ken, Japan). NLR was calculated as the ratio of neutrophils to lymphocytes. We collected data on height, body weight, body mass index, systolic blood pressure, pulse rate, Killip class, and left ventricular ejection fraction (LVEF) at admission. Type of anti-platelet therapy administered during index admission, use of beta blocker, angiotensin converting enzyme inhibitor, angiotensin receptor blocker, statin, and calcium channel blocker at discharge were also included in our data.

\section{Statistical analysis}

Continuous data are shown as mean \pm standard deviation, and categorical data are shown as number and frequency. Comparison between categorical variables was performed by Chi-square test. Differences between continuous variables were analyzed by analysis of variance and Tukey's method for hypothesis testing. Receiver operating characteristic (ROC) curves were used to set cut-off values of NLR and CRP, and patients were divided into 4 groups based on these values. Survival curves of the 4 groups at 24 months were constructed by Kaplan-Meier analysis and compared using the log-rank test. The independent predictors of adverse events were evaluated using the Cox proportional hazards model including the clinical variables associated with clinical outcomes at the level of $p<0.10$ in univariate analysis. Likelihood ratio test was used to determine whether logistic regression models that included high NLR and high CRP provided a significantly better fit than did logistic regression models limited to high NLR or high CRP alone. Statistical significance was defined as $p<0.05$. All statistical analysis was performed using IBM SPSS 22.0 (SPSS Inc., Chicago, IL, USA).

\section{Results}

A total of 467 patients were included in the study, and the median follow-up period was $27.54 \pm 14.53$ months. Patients lost to follow-up before 2 years were excluded from the analysis. Among the 467 patients, a total of 381 patients $(76.1 \%$ male, mean age $61.64 \pm 11.0$ years) with AMI who underwent $\mathrm{PCl}$ and completed 2 years of follow-up were included in the final cohort. On ROC analysis, the optimal cut-off values of NLR and CRP were the largest area under the curves predicting 24-month all cause death. The cut-off values of NLR and CRP were 6.30 (area under the ROC curve $[A U C]=0.636$, sensitivity $=34.2 \%$, specificity $=90.0 \%, p=0.007$ ) and 0.76 ( $A \cup C=0.609$, sensitivity $=53.7 \%$, specificity $=74.4 \%, p=0.044$ ), 


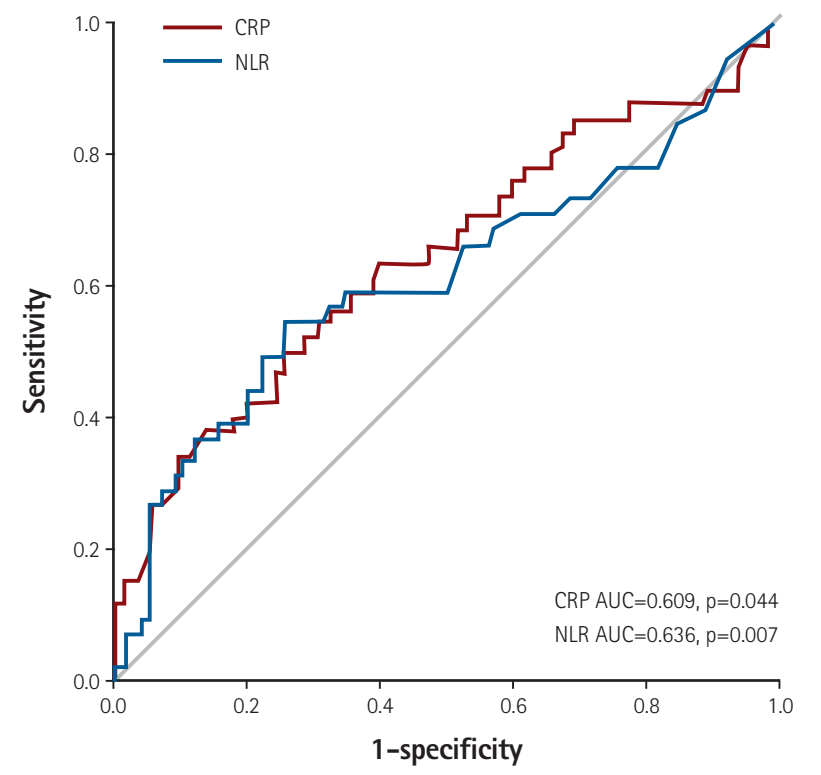

Fig. 1. ROC curves of CRP and NLR according to 24 month all-cause death. CRP: C-reactive protein, NLR: neutrophil-to-lymphocyte ratio, AUC: area under the receiver operator characteristic curve, ROC: receiver operator characteristic, MACCE: major adverse cardiac and cerebrovascular events.

respectively (Fig. 1). Positive correlation was found between NLR and CRP $(r=0.245 ; p<0.001)$. Patients were classified into 4 groups based on the optimal cut-off values of the NLR and CRP: a low NLR and a low CRP (Group 1, NLR $\leq 6.30, C R P \leq 0.76, n=249$ ), a low NLR and a high CRP (Group 2, NLR $\leq 6.30, C R P>0.76, n=84$ ), a high NLR and a low CRP (Group 3, NLR $>6.30$ and CRP $\leq 0.766, n=23$ ), a high NLR and a high CRP (Group 4, NLR $>6.30$ and CRP>0.76, $n=25$ ). Table 1 shows baseline clinical and procedural characteristics of the study population, and Table 2 shows the laboratory findings for each group. Patients in Group 4 were significantly older; had a higher Killip class, WBC, platelet-to-lymphocyte ratio (PLR), and serum creatinine; and lower LVEF, hemoglobin, and LDL-cholesterol levels compared to patients in Group 1. The proportion of patients who had diabetes mellitus (DM), chronic renal failure, and previous chronic heart failure (CHF) was higher in Group 4 compared to the other groups.

Clinical outcomes at 24-months are summarized in Table 3. Patients in Group 4 had a significantly higher rate of all-cause mortality compared with the other groups (44.0\% in Group 4 vs. 6.4\% in Group 1 vs. 13.1\% in Group 2 vs. 13\% in Group 3, p<0.001). Cardiovascular mortality ( $24.0 \%$ vs. $5.2 \%, p=0.007$ ) and MACCE (44.0\% vs. $17.3 \%, p=0.007$ ) were significantly higher in Group 4 compared with group 1 (Fig. 2). KaplanMeier analysis revealed significantly lower 24-month death-free survival in Group 4 patients (log-rank test $p<0.001$; Fig. 3).

On Cox-regression univariate analysis, age, female gender, DM,

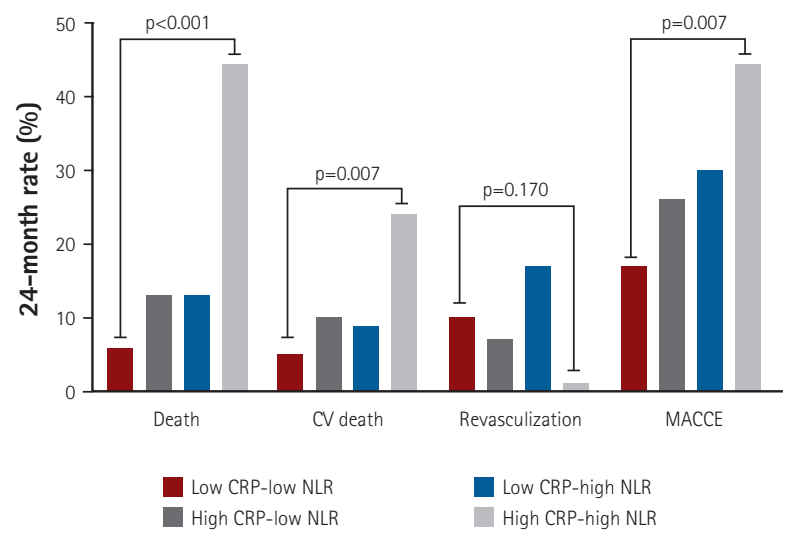

Fig. 2. Clinical outcomes in 4 groups at 24 months. Patients in the high NLR and high CRP group had significantly higher rates of all-cause mortality, cardiovascular mortality, and MACCE. The $p$ values were compared between the low NLR-low CRP group and the high NLR-high CRP group. CV: cardiovascular, CRP: C-reactive protein, NLR: neutrophilto-lymphocyte ratio, MACCE: major adverse cardiac and cerebrovascular events.

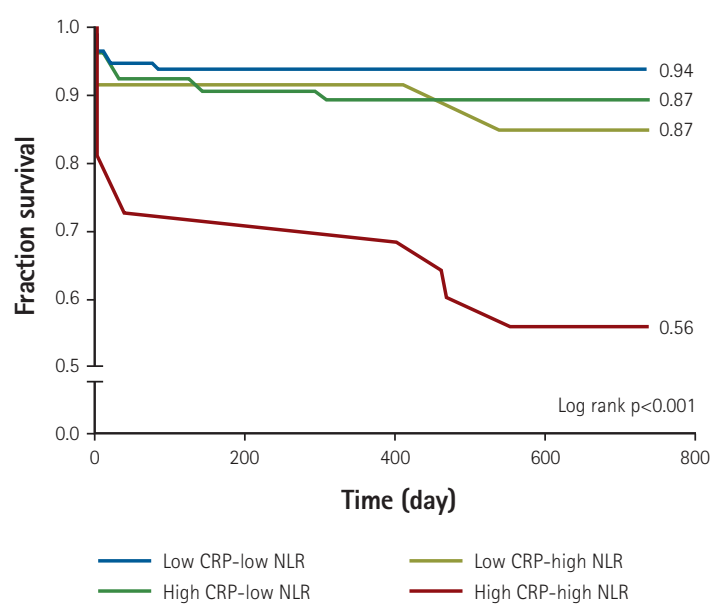

Fig. 3. Kaplan-Meier survival curve of 24 month all-cause death. Patients in the high NLR-high CRP group showed the lowest 24-month death-free survival of all groups. CRP: C-reactive protein, NLR: neutrophil-tolymphocyte ratio.

smoking, hypertension, LVEF, serum creatinine, previous CHF, NLR, CRP, and combined NLR-CRP were significant predictors of 24-month all-cause death. On multivariate analysis, a high NLR >6.30 (hazard ratio $[H R] 2.706,95 \% \mathrm{Cl} 1.055$ to $6.936, p=0.038$ ) and a high CRP $>0.76$ (HR 4.235, 95\% Cl 1.190 to $15.070, p=0.026$ ) were found to be independent predictors of 24-month all-cause death, and the combination of a high NLR and a high CRP was the strongest predictor ( $H R$ 23.172, 95\% Cl 6.575 to 81.671, $p<0.001$ ). Additional significant independent predictors were lower LVEF (HR 0.924, 95\% Cl 0.895 to 
Table 1. Baseline clinical and procedural characteristics of the study population

\begin{tabular}{|c|c|c|c|c|c|}
\hline Clinical characteristics & Low CRP-low NLR & High CRP-low NLR & Low CRP-high NLR & High CRP-high NLR & $\mathbf{p}$ \\
\hline Male & $196(78.7)$ & $64(76.2)$ & $16(69.6)$ & $71(56.0)$ & 0.071 \\
\hline Age (years) & $60.2 \pm 10.9$ & $63.6 \pm 11.4$ & $60.5 \pm 8.4$ & $65.8 \pm 10.9^{*}$ & 0.015 \\
\hline Height $(\mathrm{cm})$ & $166.2 \pm 7.9$ & $164.7 \pm 8.4$ & $164.5 \pm 5.6$ & $162.7 \pm 10.1$ & 0.074 \\
\hline Body weight (kg) & $65.9 \pm 11.3$ & $63.8 \pm 11.5$ & $62.3 \pm 7.7$ & $65.9 \pm 16.5$ & 0.248 \\
\hline BMI $\left(\mathrm{kg} / \mathrm{m}^{2}\right)$ & $23.7 \pm 2.9$ & $23.4 \pm 3.6$ & $22.9 \pm 2.5$ & $24.9 \pm 5.1$ & 0.134 \\
\hline $\operatorname{LVEF}(\%)$ & $51.0 \pm 9.0$ & $47.8 \pm 10.7$ & $46.0 \pm 10.9$ & $46.3 \pm 11.6^{*}$ & 0.005 \\
\hline Systolic BP (mmHg) & $125.4 \pm 22.1$ & $127.5 \pm 23.9$ & $117.2 \pm 22.6$ & $135.9 \pm 28.2$ & 0.162 \\
\hline Pulse rate (beat/min) & $75.0 \pm 15.9$ & $78.9 \pm 14.3$ & $77.6 \pm 22.9$ & $80.8 \pm 18.8$ & 0.260 \\
\hline Killip class & & & & & $<0.001$ \\
\hline Class 1 & $198(79.5)$ & $57(67.9)$ & $12(52.2)$ & $11(44.0)$ & \\
\hline Class 2 & $16(6.4)$ & $6(7.1)$ & 4 (17.4) & $0(0)$ & \\
\hline Class 3 & $17(6.8)$ & $15(17.9)$ & $6(26.1)$ & $11(44.0)$ & \\
\hline Class 4 & $18(7.2)$ & $6(7.1)$ & $1(4.3)$ & $3(12.0)$ & \\
\hline Diagnosis & & & & & 0.069 \\
\hline STEMI & $127(51.0)$ & 31 (36.9) & $13(56.5)$ & $9(36.0)$ & \\
\hline NSTEMI & $122(49.0)$ & $53(63.1)$ & $10(43.5)$ & $16(64.0)$ & \\
\hline Primary $\mathrm{PCl}$ & $155(62.2)$ & $33(39.3)^{*}$ & $16(69.6)^{\dagger}$ & $16(36.0)$ & $<0.001$ \\
\hline LBBB & $11(4.4)$ & $2(2.4)$ & $3(13.0)$ & $3(12.0)$ & 0.068 \\
\hline ST-segment depression & $30(12.0)$ & $18(21.4)$ & $1(4.3)$ & $6(24.0)$ & 0.042 \\
\hline Atrial fibrillation & $25(10.0)$ & $4(4.8)$ & $3(13.0)$ & $3(12.0)$ & 0.418 \\
\hline Hypertension & $125(50.2)$ & 49 (58.3) & $9(39.1)$ & $18(7.0)$ & 0.068 \\
\hline DM & 58 (23.3) & $33(39.3)^{*}$ & $10(43.5)$ & $13(52.0)^{*}$ & $<0.001$ \\
\hline Insulin & $16(6.4)$ & $14(16.7)^{*}$ & $6(26.1)^{*}$ & $2(8.0)$ & 0.002 \\
\hline Oral hypoglycemic agent & $51(20.5)$ & $30(35.7)^{*}$ & $10(43.5)$ & $10(40.0)$ & 0.003 \\
\hline Smoking & $118(47.4)$ & 33 (39.3) & 8 (34.8) & $4(16.0)^{*}$ & 0.015 \\
\hline PVD & $4(1.6)$ & $1(1.2)$ & $0(0)$ & $0(0)$ & 0.846 \\
\hline Dyslipidemia & $27(10.8)$ & 9 (10.7) & $4(17.4)$ & $2(8.0)$ & 0.753 \\
\hline CRF & $3(1.2)$ & $10(11.9)^{*}$ & $2(8.7)$ & $5(20.0)^{*}$ & $<0.001$ \\
\hline CLD & $14(5.6)$ & $8(9.5)$ & $1(4.3)$ & $2(8.0)$ & 0.609 \\
\hline Pre CHF & $14(5.6)$ & $4(4.8)$ & $2(8.7)$ & $5(20.0)^{*+}$ & 0.040 \\
\hline Pre Ml & $26(10.4)$ & $8(9.5)$ & $3(13.0)$ & $2(8.0)$ & 0.942 \\
\hline Pre PCl & $40(16.1)$ & $12(14.3)$ & $5(21.7)$ & $6(24.0)$ & 0.613 \\
\hline Pre CABG & $3(1.2)$ & $4(4.8)$ & $1(4.3)$ & $0(0)$ & 0.174 \\
\hline Family history of CAD & $24(9.6)$ & $7(8.3)$ & $4(17.4)$ & $3(12.0)$ & 0.614 \\
\hline IABP use & $17(6.8)$ & $6(7.1)$ & $6(26.1)^{* t}$ & $3(12.0)$ & 0.013 \\
\hline Gp llbllla inhibitor use & $17(6.8)$ & $5(6.0)$ & $5(21.7)^{\dagger}$ & $1(4.0)$ & 0.052 \\
\hline Procedural success & $246(98.8)$ & $81(96.4)$ & $22(95.7)$ & $20(80.0)^{*+\neq}$ & $<0.001$ \\
\hline Multi-vessel PCl & 57 (22.9) & 24 (28.6) & $9(39.1)$ & $3(12.0)$ & 0.117 \\
\hline Infarct-related artery & & & & & 0.071 \\
\hline LAD & $107(43.0)$ & $42(50.0)$ & $12(52.2)$ & $14(56.0)$ & \\
\hline LCX & $56(22.5)$ & $24(28.6)$ & $2(8.7)$ & 4 (16.0) & \\
\hline RCA & $85(34.1)$ & $18(21.4)$ & $8(34.8)$ & $7(28.0)$ & \\
\hline Left main & $1(0.4)$ & $0(0.0)$ & $1(4.3)$ & $0(0.0)$ & \\
\hline \multicolumn{6}{|l|}{ Medications } \\
\hline Antiplatelet therapy & & & & & 0.052 \\
\hline Aspirin+clopidogrel & $187(75.1)$ & 75 (89.3) & 19 (82.6) & 23 (92.0) & \\
\hline Aspirin+prasugrel & $22(8.8)$ & $3(3.6)$ & $0(0.0)$ & $1(4.0)$ & \\
\hline Aspirin+ticagrelor & 40 (16.1) & $6(7.1)$ & 4 (17.4) & $1(4.0)$ & \\
\hline Beta-blocker & 219 (88.0) & 73 (86.9) & $20(87.0)$ & 19 (76.0) & 0.416 \\
\hline ACEI & $187(75.1)$ & $55(65.5)$ & 15 (65.2) & $15(60.0)$ & 0.162 \\
\hline ARB & 43 (17.3) & 14 (16.7) & $2(8.7)$ & $8(32.0)$ & 0.179 \\
\hline Statin & $230(92.4)$ & 75 (89.3) & 22 (95.7) & $21(84.0)$ & 0.388 \\
\hline $\mathrm{CCB}$ & $19(7.6)$ & $3(3.6)$ & $2(8.7)$ & $1(4.0)$ & 0.546 \\
\hline
\end{tabular}

Values are presented as number (\%).* ${ }^{*}<0.05$ compared with low CRP, low NLR. $+p<0.05$ compared with high CRP, low NLR. $\neq p<0.05$ compared with low CRP, high NLR. ACEl: angiotensin converting enzyme inhibitor, ARB: angiotensin receptor blocker, BP: blood pressure, BMI: body mass index, CABG: coronary artery bypass surgery, CAD: coronary artery disease, CAG: coronary angiography, CCB: calcium channel blocker, CHF: chronic heart failure, CLD: chronic lung disease, CRF: chronic renal failure, CRP: C-reactive protein, DM: diabetes mellitus, IABP: intra-aortic balloon pump, LAD: left anterior descending artery, LCX: left circumflex artery, LVEF: left ventricle ejection fraction, NLR: neutrophil to lymphocyte ratio, PCl: percutaneous coronary intervention, RCA: right coronary artery 
Table 2. Baseline laboratory characteristics of the study population according to NLR and CRP

\begin{tabular}{|c|c|c|c|c|c|}
\hline \multirow{2}{*}{ Clinical characteristics } & Low CRP-low NLR & High CRP-low NLR & Low CRP-high NLR & High CRP-high NLR & \multirow{2}{*}{$\mathbf{p}$} \\
\hline & (Group 1, n=249) & (Group 2, n=84) & (Group 3, n=23) & (Group 4, n=25) & \\
\hline $\mathrm{CRP}(\mathrm{mg} / \mathrm{L})$ & $0.19 \pm 0.18$ & $3.97 \pm 3.82^{*}$ & $0.17 \pm 0.20^{+}$ & $4.39 \pm 4.03^{* \neq}$ & $<0.001$ \\
\hline WBC count (x10^9/L) & $10.14 \pm 3.22$ & $10.27 \pm 3.59$ & $12.12 \pm 3.24^{*}$ & $12.55 \pm 4.11^{*+}$ & 0.001 \\
\hline Neutrophil (\%) & $58.98 \pm 14.40$ & $65.65 \pm 9.78^{*}$ & $83.98 \pm 3.25^{*+}$ & $85.26 \pm 4.49^{*+}$ & $<0.001$ \\
\hline Lymphocyte (\%) & $31.75 \pm 13.40$ & $24.17 \pm 9.64^{*}$ & $10.07 \pm 1.55^{*+}$ & $8.50 \pm 2.83^{* \dagger}$ & $<0.001$ \\
\hline NLR & $2.40 \pm 1.46$ & $3.19 \pm 1.40^{*}$ & $8.59 \pm 1.76^{*+}$ & $11.81 \pm 5.99^{*+\neq}$ & $<0.001$ \\
\hline Platelet (x10^9/L) & $224.2 \pm 58.1$ & $231.7 \pm 76.2$ & $217.3 \pm 52.3$ & $242.1 \pm 119.5$ & 0.470 \\
\hline PLR & $8.39 \pm 4.25$ & $10.79 \pm 4.86^{*}$ & $22.21 \pm 7.46^{*+}$ & $32.54 \pm 22.49^{*}+\neq$ & $<0.001$ \\
\hline Hemoglobin (mg/dL) & $13.92 \pm 1.99+$ & $12.847 \pm 2.48^{*}$ & $13.98 \pm 1.75^{\dagger}$ & $11.50 \pm 2.06^{* \neq}$ & $<0.001$ \\
\hline AST (U/L) & $59.69 \pm 124.8$ & $85.04 \pm 100.3$ & $98.52 \pm 198.2$ & $87.40 \pm 118.9$ & 0.208 \\
\hline $\operatorname{ALT}(\mathrm{U} / \mathrm{L})$ & $40.66 \pm 104.9$ & $45.05 \pm 69.57$ & $37.39 \pm 34.5$ & $50.60 \pm 57.26$ & 0.937 \\
\hline CK-MB peak (ng/dL) & $120.8 \pm 111.1$ & $82.79 \pm 100.1^{*}$ & $150.5 \pm 122.1^{\dagger}$ & $100.4 \pm 111.8$ & 0.015 \\
\hline Tnl peak (ng/dL) & $35.49 \pm 36.67$ & $28.89 \pm 35.06$ & $50.31 \pm 39.56$ & $32.42 \pm 35.55$ & 0.097 \\
\hline $\mathrm{SCr}(\mathrm{mg} / \mathrm{dL})$ & $0.99 \pm 0.58$ & $1.57 \pm 1.49^{*}$ & $1.19 \pm 1.59$ & $2.92 \pm 2.94^{*+\neq}$ & $<0.001$ \\
\hline Total cholesterol (mg/dL) & $175.2 \pm 44.4$ & $176.4 \pm 58.3$ & $165.4 \pm 50.8$ & $150.5 \pm 44.6$ & 0.104 \\
\hline HDL cholesterol (mg/dL) & $43.23 \pm 10.75$ & $38.95 \pm 11.04^{*}$ & $44.76 \pm 10.30$ & $41.38 \pm 16.07$ & 0.019 \\
\hline Triglyceride (mg/dL) & $117.4 \pm 81.9$ & $110.4 \pm 64.5$ & $101.3 \pm 72.3$ & $128.8 \pm 81.2$ & 0.619 \\
\hline LDL cholesterol (mg/dL) & $111.2 \pm 41.2$ & $108.6 \pm 38.6$ & $99.3 \pm 44.9$ & $83.4 \pm 44.7^{*}$ & 0.021 \\
\hline Glucose (mg/dL) & $171.8 \pm 81.8$ & $173.9 \pm 73.7$ & $201.1 \pm 87.4$ & $214.2 \pm 114.7$ & 0.046 \\
\hline HbAlc & $6.29 \pm 1.10$ & $6.55 \pm 1.52$ & $6.92 \pm 2.03$ & $6.86 \pm 1.47$ & 0.031 \\
\hline
\end{tabular}

${ }^{*} p<0.05$ compared with low CRP, low NLR. ${ }^{+} p<0.05$ compared with high CRP, low NLR. ${ }^{\ddagger} p<0.05$ compared with low CRP, High NLR. ALT: alanine transaminase, AST: aspartate transaminase, CK-MB: creatinine kinase-myocardial band isoenzyme, CRP: C-reactive protein, HDL: high-density lipoprotein, LDL: low-density lipoprotein, NLR: neutrophil to lymphocyte ratio, SCr: serum creatinine, Tnl: troponin I

Table 3. Clinical outcomes of the study population at 24 months according to NLR and CRP

\begin{tabular}{|c|c|c|c|c|c|}
\hline \multirow{2}{*}{ Clinical outcomes } & \multirow{2}{*}{$\begin{array}{l}\text { Low CRP-low NLR } \\
\text { (Group 1, n=249) }\end{array}$} & \multirow{2}{*}{$\begin{array}{c}\text { High CRP-low NLR } \\
\text { (Group 2, n=84) }\end{array}$} & \multirow{2}{*}{$\begin{array}{c}\text { Low CRP-high NLR } \\
\text { (Group 3, n=23) }\end{array}$} & \multirow{2}{*}{$\begin{array}{c}\text { High CRP-high NLR } \\
\text { (Group 4, n=25) }\end{array}$} & \multirow{2}{*}{$\mathbf{p}$} \\
\hline & & & & & \\
\hline Death & $16(6.4)$ & $11(13.1)$ & $3(13.0)$ & $11(44.0)^{*+\neq}$ & $<0.001$ \\
\hline Cardiovascular death & $13(5.2)$ & $8(9.5)$ & $2(8.7)$ & $6(24.0)^{*}$ & 0.007 \\
\hline Recurrence of AMI & $5(2.0)$ & $3(3.6)$ & $2(8.7)$ & $0(0.0)$ & 0.199 \\
\hline Cerebrovascular accident & $3(1.2)$ & $4(4.8)$ & $0(0.0)$ & $0(0.0)$ & 0.143 \\
\hline Stent thrombosis & $4(1.6)$ & $1(1.2)$ & $2(8.7)$ & $0(0.0)$ & 0.081 \\
\hline Revascularization & $25(10.0)$ & $6(7.1)$ & $5(17.4)$ & $0(0.0)$ & 0.170 \\
\hline TLR & $6(2.4)$ & $2(2.4)$ & $2(8.7)$ & $0(0.0)$ & 0.256 \\
\hline TVR & $13(5.2)$ & $3(3.6)$ & $3(13.0)$ & $0(0.0)$ & 0.183 \\
\hline MACCE & 43 (17.3) & $22(26.2)$ & 7 (30.4) & $11(44.0)^{*}$ & 0.007 \\
\hline
\end{tabular}

Values are presented as number (\%). ${ }^{*} p<0.05$ compared with low CRP, low NLR. $+p<0.05$ compared with high CRP, low NLR. $\neq p<0.05$ compared with low CRP, high NLR. CRP: C-reactive protein, NLR: neutrophil to lymphocyte ratio, AMI: acute myocardial infarction, TLR: target lesion revascularization, TVR: target vessel revascularization, MACCE: major adverse cardiac and cerebrovascular events

0.954, $p<0.001)$, DM (HR 3.791, 95\% Cl 1.831 to $7.846, p<0.001)$, and smoking (HR 0.302, 95\% Cl 0.114 to $0.802, p=0.016$ ) (Table 4). The combination of a high NLR and a high CRP did not predict 24-month MACCE (Table 5).

In the likelihood ratio test comparing the fit of prediction models using high NLR and high CRP to the fit of models using high NLR or high CRP alone, the assessment of both parameters provided a significantly improved ability to predict risk. Models including both high NLR and high CRP provided a significant improvement in prediction $(p<0.001)$ compared with models including only high NLR or high CRP. 
Table 4. Predictors of 24 month all-cause death by multivariate Cox regression analysis

\begin{tabular}{|c|c|c|c|c|}
\hline \multirow{2}{*}{ Variables } & \multicolumn{4}{|c|}{ Hazard ratio ( $95 \%$ confidence interval) } \\
\hline & No adjustment & $\mathbf{p}$ & Adjustment & $p$ \\
\hline Age & $1.063(1.028-1.099)$ & $<0.001$ & $1.018(0.977-1.061)$ & 0.396 \\
\hline Female & $3.296(1.786-6.081)$ & $<0.001$ & $1.593(0.720-3.525)$ & 0.250 \\
\hline Diabetes mellitus & $4.544(2.405-8.587)$ & $<0.001$ & $3.791(1.831-7.846)$ & $<0.001$ \\
\hline Smoking & $0.172(0.067-0.437)$ & $<0.001$ & $0.302(0.114-0.802)$ & 0.016 \\
\hline Serum creatinine & $1.314(1.162-1.485)$ & $<0.001$ & $1.126(0.971-1.306)$ & 0.115 \\
\hline Hypertension & $2.245(1.146-4.399)$ & 0.018 & $1.563(0.686-3.563)$ & 0.288 \\
\hline LVEF & $0.914(0.886-0.944)$ & $<0.001$ & $0.924(0.895-0.954)$ & $<0.001$ \\
\hline Previous CHF & $2.583(1.086-6.143)$ & 0.032 & $0.752(0.261-2.168)$ & 0.598 \\
\hline $\mathrm{CRP}>0.76$ & $3.101(1.678-5.733)$ & $<0.001$ & $2.706(1.055-6.936)$ & 0.038 \\
\hline$N L R>6.3$ & $1.965(1.423-2.714)$ & $<0.001$ & 4.235 (1.190-15.070) & 0.026 \\
\hline $\mathrm{NLR}>6.3 \& \mathrm{CRP}>0.76$ & $5.566(2.789-11.111)$ & $<0.001$ & $23.172(6.575-81.671)$ & $<0.001$ \\
\hline
\end{tabular}

Adjusted for age, gender, diabetes mellitus, smoking, serum creatinine, hypertension, LVEF, previous CHF, NLR, and CRP. LVEF: left ventricular ejection fraction, CHF: chronic heart failure, CRP: C-reactive protein, NLR: neutrophil to lymphocyte ratio

Table 5. Predictors of 24-month MACCE by multivariate Cox regression analysis

\begin{tabular}{|c|c|c|c|c|}
\hline \multirow{2}{*}{ Variables } & \multicolumn{4}{|c|}{ Hazard ratio (95\% confidence interval) } \\
\hline & No adjustment & $\mathbf{p}$ & Adjustment & $\mathbf{p}$ \\
\hline Age & $1.032(1.010-1.054)$ & 0.004 & $1.003(0.978-1.029)$ & 0.794 \\
\hline Female & $2.029(1.296-3.176)$ & 0.002 & $1.295(0.771-2.175)$ & 0.328 \\
\hline Diabetes mellitus & $2.908(1.889-4.476)$ & $<0.001$ & $2.642(1.670-4.181)$ & $<0.001$ \\
\hline Smoking & $0.547(0.344-0.870)$ & 0.112 & $0.643(0.399-1.035)$ & 0.069 \\
\hline Serum creatinine & $1.185(1.051-1.336)$ & 0.006 & $1.048(0.904-1.215)$ & 0.536 \\
\hline Hypertension & $1.429(0.921-2.218)$ & 0.112 & $1.024(0.629-1.669)$ & 0.923 \\
\hline LVEF & $0.944(0.922-0.966)$ & $<0.001$ & $0.950(0.929-0.973)$ & $<0.001$ \\
\hline Previous CHF & $1.441(0.664-3.126)$ & 0.355 & $0.685(0.288-1.629)$ & 0.392 \\
\hline $\mathrm{CRP}>0.76$ & $1.886(1.214-2.929)$ & 0.005 & $1.461(0.903-2.362)$ & 0.123 \\
\hline$N L R>6.3$ & 1.461 (1.125-1.897) & 0.004 & 1.189 (0.894-1.580) & 0.234 \\
\hline$N L R>6.3 \&$ CRP $>0.76$ & 2.331 (1.236-4.398) & 0.009 & $1.081(0.335-3.487)$ & 0.896 \\
\hline
\end{tabular}

Adjusted for age, gender, diabetes mellitus, smoking, serum creatinine, hypertension, LVEF, previous CHF, NLR, and CRP. LVEF: left ventricle ejection fraction, CHF: chronic heart failure, CRP: C-reactive protein, NLR: neutrophil to lymphocyte ratio

\section{Discussion}

In the present analysis of $381 \mathrm{AMl}$ patients who underwent $\mathrm{PCl}$, we found that increased levels of the combined parameters of NLR and CRP on admission were associated with increased risk of long-term mortality and MACCE compared with lower NLR and CRP. We also found that the elevation of both NLR and CRP on admission was the strongest independent predictor of all cause death during 24 months of follow-up. To our knowledge, this is the first analysis investigating the value of combined NLR and CRP to predict long-term prognosis in AMl patients undergoing PCl.
Due to the growing attention in recent years on the effects of inflammatory status on initiation and progression of infarction in the myocardium, many studies have focused on the relations between inflammatory biomarkers and adverse clinical outcomes in patients with AMI. The results of this study are in agreement with recently published data that showed an association between higher NLR or CRP and poor clinical outcomes in patients with AMI.411122) Several researchers have found that it is possible to predict the progression and prognosis of AMI with NLR or CRP, but integrated use of NLR and CRP as a predictor of AMI has not been investigated in previous studies. 


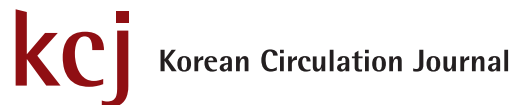

A recent study revealed that high NLR is closely related with the increased risk of adverse cardiac events, ${ }^{13)}$ and it aroused major interest in the association between elevated NLR and poor prognosis in AMI patients. One of the possible hypotheses of this association is the role of neutrophils as a mediator of inflammatory response to acute damage of myocardium ${ }^{14)}$ because they release several biochemical materials such as reactive oxygen species and proteolytic enzymes. ${ }^{15)}$ On the other hand, lymphocytes are decreased as a result of apoptosis caused by inflammation. ${ }^{16)}$ Consequently, high NLR suggests further risk of infarction of the myocardium. CRP is also a biomarker that indicates an inflammatory status in vivo. Previous studies have found that CRP can be utilized as a meaningful prognostic marker in patients with AMI. ${ }^{17)}$ A possible hypothesis that CRP can help assess the prognosis of MI patients can be explained by the relationship between elevated CRP and active inflammatory reaction that results in plaque instability. ${ }^{18) 19)}$

A recent study has suggested that reduced inflammation as evidenced by neutrophil count and CRP was quite strongly associated with reduced infarct size in patients with STEMI undergoing primary $\mathrm{PCl}$ and who were treated with the anti-inflammatory drug colchine. ${ }^{20)}$ The researchers obtained blood samples at baseline (on admission), every 4 hours for the first 72 hours post-presentation, and every 12 hours thereafter and compared the peak values of neutrophil count and CRP. Both infarct size and plaque vulnerability were shown to be important and independent predictors for clinical outcomes. The pathophysiology of the values of NLR and CRP according to obtained time after an ACS event, especially in STEMI, may be quite different. We performed one blood sampling shortly before the index procedure in our subjects regardless of their presentation. Therefore, the values of NLR and CRP in the present study may be heterogeneous from a pathophysiologic point of view, especially in STEMI patients. Moreover, some anti-inflammatory drugs might have affected the results of our study.

$\mathrm{CBC}$ is routinely performed in almost all patients on admission. It is inexpensive, and the results can be obtained within about 30 minutes. Because NLR and CRP can be easily obtained from routine laboratory examination in patients visiting the hospital due to acute chest pain, prediction of the prognosis in AMI patients with these biomarkers might be much easier and more comfortable than other biomarkers. PLR is also known as a good prognostic marker for cardiovascular events. ${ }^{2122)}$ In a previous study, combined NLR and PLR levels also predicted severity of $\mathrm{CAD}^{23)}$ and adverse clinical outcomes. ${ }^{24)}$ In our study, PLR was also a good predictor for all-cause death during 24 months of follow-up, and the AUC of PLR was similar to that of combined NLR and CRP. We also found a significant positive correlation between PLR and combined NLR and CRP. Although we investigated whether combined use of NLR and CRP better predict clinical outcomes than NLR or CRP only, these two parameters had already been described as predictors of worse cardiovascular outcomes in CVD patients in several studies; therefore, one can argue that the combination of these two biomarkers is not new. However, we thought that the combined use of the two parameters could guide physicians to make a better clinical decision if better predictability was demonstrated for the combined parameters than the individual parameters in AMI patients undergoing $\mathrm{PCl}$. If these values are used in addition to the existing models to monitor the prognosis in AMI patients, the efficiency and accuracy of those models will increase. Moreover, a better preemptive plan and treatment course with more definite directions will contribute to a superior treatment effect, and patient compliance may improve.

Although all-cause death is a major clinical outcome of the study, it has been generally accepted that the 2-year MACCE is also an important clinical outcome. Our results show that the combined use of a high NLR and a high CRP did not predict 24-month MACCE. This finding can be explained as follows. First, larger populations and further follow-up are required to evaluate the usefulness of combined NLR and CRP. The number of patients in Group 4 was too small to evaluate and compare MACCE and each event during the 2 years among the groups. Second, we determined the optimal cutoff values of NLR and CPR for predicting 2-year all-cause mortality, not for predicting 2-year MACCE. To find the optimal cut-off values of these parameters for good prediction, the ROC curves of 2-year all-cause mortality and MACCE were compared. The AUC of the ROC curve of NLR and CRP with 2-year MACCE was smaller than that with 2-year all-cause mortality and was not statistically significant (Supplementary Fig. 1 in the online-only Data Supplement). Therefore, we thought it would be better to set the cut-off value according to the all-cause mortality in order to predict cardiovascular outcomes.

One interesting finding in our study is that patients with low NLRlow CRP were more likely to smoke than those in the other groups. We considered this to be an instance of the 'smoking paradox'. More than 25 years ago, the 'smoking paradox' was introduced into the scientific literature according to the observations that smokers with AMI had lower mortality than non-smokers. ${ }^{25-28)}$ However, this conclusion remains controversial because some other findings after the advent of the "smoking paradox" did not support this hypothesis. ${ }^{29)}$

There is need for additional research before these markers can be used as an indicator of poor outcomes of AMI in clinical situations. First, the normal range of NLR in different races has not been explored, and the normal ranges of absolute neutrophil and lymphocyte counts have not been investigated. A recent study revealed that there is a racial difference in NLR in patients with NSTEMI. ${ }^{30)}$ Also, the pathophysiologic role of NLR on AMI has yet to be revealed. Whether the NLR is merely the outcome of poor prognosis of AMI patients or 
if it truly plays an important pathognomonic role for development of MI has not been established, and further studies are needed to supplement these unidentified aspects.

\section{Study limitations}

Our study has several limitations that should be considered. First, although we analyzed a consecutive series of a large number of AMI patients in this study, the observation was performed in a single tertiary center in one locality. Therefore, selection bias could exist in this study. Moreover, there were marked differences in baseline demographic and clinical characteristics according to biomarker status. Some of the baseline parameters were not balanced between the groups, which could have weakened the results of our study. Although we attempted to adjust for several clinical factors, the possibility of residual unmeasured confounding effects remains. Further studies with a larger sample size are needed. Second, more than $10 \%(n=86)$ of the initially enrolled patients were lost to follow-up. Third, because we used NLR and CRP values measured at admission before $\mathrm{PCl}$ to assess their combined potency in predicting prognosis, this study cannot suggest the aspect of transition of NLR and CRP. It might be very useful to analyze the trends of NLR and CRP over time and determine if they remain as predictors of CAD severity. However, a previous study showed that there is a significant correlation between long-term mortality and any NLR (measured at initial presentation, last in the index admission , maximum, or average NLR). ${ }^{111}$ Fourth, we did not compare the prognostic values of NPR and CRP with other markers such as $\mathrm{N}$-terminal pro-B-type natriuretic peptide, fibrinogen, or myeloperoxidase. Finally, the sample sizes of the low CRP-high NLR group $(n=23)$ and high NLR-CRP group $(n=25)$ were too small for statistical comparison to the other groups. Given our modest total sample size, our data should not lead to a definitive conclusion and need to be confirmed in future studies.

In conclusion, the combined values of NLR and CRP are a valuable and meaningful tool to predict long-term mortality in AMI patients who have undergone PCI. Further studies are needed to supplement the results of our study. Nevertheless, the results of our study add to the growing body of literature evidence that the combined parameters of NLR and CRP measured before $\mathrm{PCl}$ might be used as a useful indicator of adverse outcomes in AMI patients.

\section{Acknowledgements}

This work was conducted on behalf of Student Inje Medical Research course. The authors thank students of University of Inje College of Medicine, Jee-Woo Kim, Ha-Eun Jo, Gi-Rim Kim, Min-Ki Lee, Sung-Jin
Hwang, Ju-Yung Jeong and Young-in Choi, for their assistance with data collection.

\section{Supplementary Material}

The online-only Data Supplement is available with article at https://doi.org/10.4070/kcj.2016.0327.

\section{References}

1. Ross R. Atherosclerosis--an inflammatory disease. N Engl J Med 1999:340:115-26.

2. Libby P, Ridker PM, Hansson GK; Leducq Transatlantic Network on Atherothrombosis. Inflammation in atherosclerosis: from pathophysiology to practice. J Am Coll Cardio/ 2009;54:2129-38.

3. Ogita M, Miyauchi $K$, Tsuboi $S$, et al. Impact of combined C-reactive protein and high-density lipoprotein cholesterol levels on long-term outcomes in patients with coronary artery disease after a first percutaneous coronary intervention. Am J Cardio/ 2015;116:999-1002.

4. Han YC, Yang TH, Kim DI, et al. Neutrophil to lymphocyte ratio predicts long-term clinical outcomes in patients with ST-segment elevation myocardial infarction undergoing primary percutaneous coronary intervention. Korean Circ J 2013;43:93-9.

5. Gibson PH, Croal BL, Cuthbertson BH, et al. Preoperative neutrophillymphocyte ratio and outcome from coronary artery bypass grafting. Am Heart J 2007;154:995-1002.

6. Gibson $\mathrm{PH}$, Cuthbertson $\mathrm{BH}$, Croal BL, et al. Usefulness of neutrophil/ lymphocyte ratio as predictor of new-onset atrial fibrillation after coronary artery bypass grafting. Am J Cardio/ 2010;105:186-91.

7. Nakamura T, Matsumine A, Matsubara T, Asanuma K, Uchida A, Sudo A. The combined use of the neutrophil-lymphocyte ratio and C-reactive protein level as prognostic predictors in adult patients with soft tissue sarcoma. J Surg Onco/ 2013;108:481-5.

8. O'Gara PT, Kushner FG, Ascheim DD, et al. 2013 ACCF/AHA guideline for the management of ST-elevation myocardial infarction: a report of the American College of Cardiology Foundation/American Heart Association task force on practice guidelines. Circulation 2013;127:e362-425.

9. Amsterdam EA, Wenger NK, Brindis RG, et al. 2014 AHA/ACC guideline for the management of patients with non-ST-elevation acute coronary syndromes: a report of the American College of Cardiology/American Heart Association task force on practice guidelines. J Am Coll Cardiol 2014;64:e139-228.

10. Cutlip DE, Windecker $S$, Mehran $R$, et al. Clinical end points in coronary stent trials: a case for standardized definitions. Circulation 
2007:115:2344-51.

11. Azab B, Zaher M, Weiserbs $K F$, et al. Usefulness of neutrophil to lymphocyte ratio in predicting short- and long-term mortality after non-ST-elevation myocardial infarction. Am J Cardiol 2010;106:470-6.

12. Tamhane UU, Aneja S, Montgomery D, Rogers EK, Eagle KA, Gurm HS. Association between admission neutrophil to lymphocyte ratio and outcomes in patients with acute coronary syndrome. Am J Cardio/ 2008;102:653-7.

13. Fowler AJ, Agha RA. Neutrophil/lymphocyte ratio is related to the severity of coronary artery disease and clinical outcome in patients undergoing angiography--the growing versatility of NLR. Atherosclerosis 2013;228:44-5.

14. Keeley EC, Boura JA, Grines CL. Primary angioplasty versus intravenous thrombolytic therapy for acute myocardial infarction: a quantitative review of 23 randomised trials. Lancet 2003;361:13-20.

15. Forman MB, Puett DW, Virmani R. Endothelial and myocardial injury during ischemia and reperfusion: pathogenesis and therapeutic implications. J Am Coll Cardiol 1989;13:450-9.

16. Alpert JS, Thygesen K, Antman E, Bassand JP. Myocardial infarction redefined--a consensus document of the joint european society of cardiology/american college of cardiology committee for the redefinition of myocardial infarction. J Am Coll Cardiol 2000;36:959-69.

17. De Servi S, Mariani M, Mariani G, Mazzone A. C-reactive protein increase in unstable coronary disease cause or effect? J Am Coll Cardiol 2005;46:1496-502.

18. Hansson GK, Libby P, Tabas I. Inflammation and plaque vulnerability. J Intern Med 2015;278:483-93.

19. Agrawal A, Gang TB, Rusiñol AE. Recognition functions of pentameric C-reactive protein in cardiovascular disease. Mediators Inflamm 2014;2014:319215.

20. Deftereos S, Giannopoulos G, Angelidis C, et al. Anti-inflammatory treatment with colchicine in acute myocardial infarction: a pilot study. Circulation 2015;132:1395-403.

21. Ugur M, Gul M, Bozbay M, et al. The relationship between platelet to
Iymphocyte ratio and the clinical outcomes in ST elevation myocardial infarction underwent primary coronary intervention. Blood Coagul Fibrinolysis 2014;25:806-11.

22. Temiz A, Gazi E, Güngör Ö, et al. Platelet/lymphocyte ratio and risk of in-hospital mortality in patients with ST-elevated myocardial infarction. Med Sci Monit 2014;20:660-5.

23. Sari I, Sunbul M, Mammadov C, et al. Relation of neutrophil-tolymphocyte and platelet-to-lymphocyte ratio with coronary artery disease severity in patients undergoing coronary angiography. Kardiol Pol 2015;73:1310-6.

24. Cho Kl, Ann SH, Singh GB, Her AY, Shin ES. Combined usefulness of the platelet-to-lymphocyte ratio and the neutrophil-to-lymphocyte ratio in predicting the long-term adverse events in patients who have undergone percutaneous coronary intervention with a drugeluting stent. PLoS One 2015;10:e0133934.

25. Helmers C. Short and long-term prognostic indices in acute myocardial infarction. A study of 606 patients initially treated in a coronary care unit. Acta Med Scand Supp/ 1973;555:7-26.

26. Kelly TL, Gilpin E, Ahnve S, Henning H, Ross J Jr. Smoking status at the time of acute myocardial infarction and subsequent prognosis. Am Heart J 1985;110:535-41.

27. Sparrow D, Dawber TR. The influence of cigarette smoking on prognosis after a first myocardial infarction. A report from the Framingham study. J Chronic Dis 1978;31:425-32.

28. Weinblatt $E$, Shapiro S, Frank CW, Sager RV. Prognosis of men after first myocardial infarction: mortality and first recurrence in relation to selected parameters. Am J Public Health Nations Health 1968;58:1329-47.

29. Aune E, Røislien J, Mathisen M, Thelle DS, Otterstad JE. The "smoker's paradox" in patients with acute coronary syndrome: a systematic review. BMC Med 2011;9:97.

30. Misumida N, Kobayashi A, Kanei Y. Racial differences in the neutrophil-to-lymphocyte ratio in patients with non-ST-segment elevation myocardial infarction. Coron Artery Dis 2015;26:381-5. 